\title{
EL IMPACTO DE LA MENTORÍA ENTRE PROFESORES UNIVERSITARIOS EN PROGRAMAS DE APRENDIZAJE-SERVICIO
}

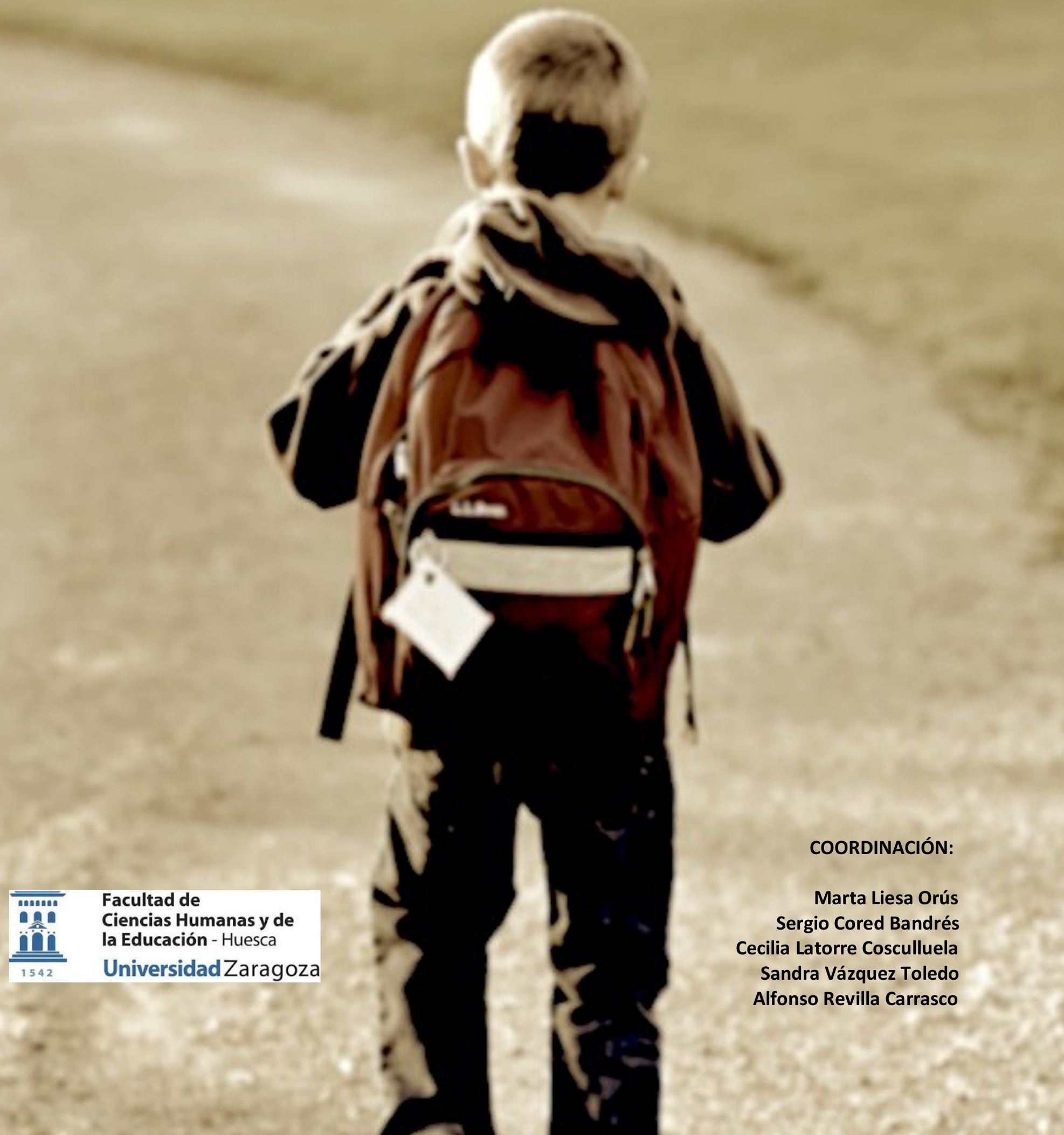


Copyright $\odot$ los autores

(c) Diseño de portada: Sergio Cored Bandrés

El impacto de la mentoría entre profesores universitarios en programas de aprendizaje-servicio

Para citar la obra:

Liesa, M., Cored, S., Latorre, C., Vázquez, S., y Revilla, A. (2019). El impacto de la mentoría entre profesores universitarios en programas de aprendizaje-servicio. España, Zaragoza: Prensas

Universitarias de Zaragoza.

Coordinadores: Marta Liesa, Sergio Cored, Cecilia Latorre, Sandra Vázquez y Alfonso Revilla.

Edita: Prensas Universitarias de la Universidad de Zaragoza

Universidad de Zaragoza

Huesca, 2019

ISBN: 978-84-09-15281-0

Depósito Legal:

Reservados todos los derechos. Cualquier forma de reproducción, distribución, comunicación pública o transformación de esta obra solo puede ser realizada con la autorización de sus titulares, salvo excepción prevista por la ley. Diríjase a CEDRO (Centro Español de Derechos Reprográficos, www.cedro.org) si necesita fotocopiar o escanear algún fragmento de esta obra.

(C) $(1) \Theta$ 
La metodología de aprendizaje-servicio (ApS) nos permite no solo trabajar con los alumnos aspectos académicos o curriculares, sino también, las partes social y moral posibilitando un aprendizaje global basado en la experiencia e imprescindible para la formación de buenos maestros.

Por otro lado, además del alumnado, las experiencias ApS repercuten también en los docentes de una forma significativa, implicándoles de una manera directa y permitiéndoles sentirse satisfechos como profesionales al percibir cómo el alumnado considera útil lo aprendido dentro del aula. Por último, y como esencia de esta metodología, cabe destacar la gran ayuda que se presta a las entidades sociales con las que se trabaja, facilitando su labor y dándoles más visibilidad dentro de la sociedad. 


\section{ÍNDICE}

Introducción Pilar Arranz y Marta Liesa

Capítulo 1. ¿Cómo podemos promover la práctica de actividad física en poblaciones desfavorecidas? "Pío se mueve": un diseño de intervención

María Sanz-Remacha, Alberto Aibar Solana, Javier Sevil-Serrano, Ángel Abós, Luis García-González.

Capítulo 2. Del ojo a la imagen; proyecto de educación plástica y visual par la formación de maestros, con niños con síndrome de down

Alfonso Revilla, Fernando J. Sadio-Ramos, Ana Lacarte, Sara Ledesma y Silke Klabbers

Capítulo 3. Evaluación del programa de mentoría de ApS para profesores universitarios en la Facultad de Ciencias Humanas y de la Educación

Cecilia Latorre Cosculluela, Marta Liesa Orús, Sandra Vázquez Toledo y Sergio Cored Bandrés

Capítulo 4. Nos vamos de minimarcha con Aspace (experiencia de ApS con estudiantes de magisterio)

Ma Pilar Otal Piedrafita, Natalia Sobradiel, Marta Liesa Orús Ester Ayllón Negrillo y Conrado Salinas Altemir

Capítulo 5. Propuesta de un programa de apoyo extraescolar basado en actividades de aprendizaje lúdicas que permitan desarrollar las competencias de dos asignaturas del grado de magisterio llevando a cabo la implementación de prácticas de ApS en un centro educativo

Ana Guillén Ubico y Silvia Anzano Oto

Capítulo 6. Proyecto de ApS de estudiantes CPIFP Montearagón con los niños de madres en riesgo de exclusión social del proyecto "Cuidado entre madres" de Cáritas Diocesana

Cristina Sierco Jarque y Marta Liesa Orús

Capítulo 7. Um día en el mercado...nota sobre una experiencia interdisciplinar

J. Fernando Sadio-Ramos, María Angustias Ortiz-Molina, Carmen MartínezSamper

Capítulo 8. Visión del alumnado universitario sobre una experiencia de ApS: pisos de vida independiente

Sergio Cored Bandrés, Marta Liesa Orús, Cecilia Latorre Cosculluela y Sandra Vázquez Toledo

Capítulo 9. Campus oportunidades para la inclusión (convivencia, aprendizaje colaborativo e inclusión): una experiencia de aprendizaje servicio

Elías Vived Conte y Jorge Arbués Salazar

Capítulo 10. El aprendizaje servicio: una oportunidad para el incremento de la formación profesional y el crecimiento personal de los estudiantes

Rafael Díaz Fernández, María Sierra Berdejo y Anabel Satué Orós

Capítulo 11. Buenas prácticas docentes que promueven el desarrollo de conductas prosociales durante los recreos cooperativos e inclusivos

Ana María Mega Avellaneda y Marta Liesa Orús

Capítulo 12. ITB: un Proyecto de Aprendizaje-Servicio en la ciudad de Huesca Javier Rodrigo-Sanjoaquín, Gemma Bermejo-Martínez y Eduardo Generelo Lanaspa 
Capítulo 13. La influencia de la inteligencia emocional en la ansiedad. La depresión y las quejas somáticas en niños de educación infantil en situaciones de vulnerabilidad. El efecto modulador del sexo

Ana Mateo Folgado y Eva M. Lira Rodríguez

Capitulo 14: La universidad construye y transforma la ciudad desde/con las niñas y los niños de Huesca como experiencia de aps

Annabella Salamanca Villate, Ester Ayllón Negrillo, Azucena Lozano Roy, $\mathbf{M}^{a}$ Ángeles Eito Más, Silvia Martínez Olivas, Lidia Bañares Vázquez y Mª Pilar Moreno Rodríguez 


\section{CAPÍTULO 7}

\section{UM DÍA EN EL MERCADO... NOTA SOBRE UNA EXPERIENCIA INTERDISCIPLINAR}

Sadio-Ramos, Fernando J. ${ }^{1}$; Ortiz-Molina, María Angustias²; Martínez-Samper, Carmen ${ }^{3}$

(1) Escola Superior de Educação do Instituto Politécnico de Coimbra; Grupo de Investigación HUM-672 AREA (Universidad de Granada); Unidad de Investigación IEF (Universidad de Coimbra), framos@esec.pt, (2) Grupo de Investigación HUM-672 AREA (Universidad de Granada), maortiz@ugr.es, (3) Facultad de Ciencias Sociales y Humanas de Teruel. Grado en Bellas Artes. Universidad de Zaragoza, casamper@unizar.es

\section{RESUMEN}

El texto trata de una actividad interdisciplinar desarrollada dentro de un proyecto de intervención sociocultural a través de Expresión Dramática con personas mayores. Tiene lugar en la Escuela Superior de Educación del Instituto Politécnico de Coimbra (ESE-IPC), en la que se creó el Club de Expresión Dramática "Os Putos" ["Los Chicos"]. El Club tiene su origen en el Proyecto de innovación pedagógica SeCApS Sostenibilidad Curricular y Aprendizaje en Servicio. En su ámbito, se llevó a cabo una experiencia pedagógica en el curso de 2014-15, que posibilitó la creación del Club en diciembre de 2015.

Desde entonces, sus miembros han desarrollado la práctica estética de la Expresión Dramática, dando origen a un portafolio de piezas y performances públicas.

Haremos una presentación enumerando algunas de las actividades desarrolladas por el Club desde su creación.

La última representación, Un día en el Mercado, ha integrado en su desarrollo una contribución interdisciplinar de las artes plásticas, a cargo de la tercera firmante de la comunicación. Durante su estancia Erasmus+ en ESE-IPC, se ha asociado al trabajo en curso, diseñando una presentación y un escenario digital para enriquecer y reforzar la presentación pública de la pieza.

Palabras Clave: ApS - Aprendizaje en Servicio; educación no formal; expresión dramática con séniores

\section{ABSTRACT}

The text presents an interdisciplinary activity developed within a project of sociocultural intervention through Dramatic Expression with older people. It takes place in the College of Education of the Polytechnic of Coimbra, in which the Dramatic Expression Club "The Kids" was created.

The Club has its origins in the Pedagogical Innovation Project SeCApS [Curricular Sustainability and Service Learning]. In its scope, a pedagogical experience was carried out in the course of 2014-15, which made possible the creation of the Club in December 2015.

Since then, its members have developed the aesthetic practice of Dramatic Expression, giving rise to a portfolio of pieces and public performances.

We will make a presentation listing some of the activities developed by the Club since its inception.

The last representation, $A$ Day in the Market, has integrated in its development an interdisciplinary contribution of the plastic arts, in charge of the third signatory of the communication. During her Erasmus + mission at College of Education of the Polytechnic of Coimbra, she has joined the work in progress, designing a presentation and a digital scenario to enrich and reinforce the public presentation of the piece.

Keywords: Dramatic Expression with Older People; Non-formal Education; Service Learning 


\section{INTRODUCCIÓN}

El texto trata sobre todo de una actividad interdisciplinar que hemos desarrollado dentro de un proyecto de intervención sociocultural a través de Expresión Dramática con personas mayores. El proyecto viene siendo desarrollado en la Escuela Superior de Educación del Instituto Politécnico de Coimbra (ESE-IPC). Esta institución alberga un proyecto de educación no formal, de intervención comunitaria: la Escuela de Educación Sénior, promovida por una organización no gubernamental, el Instituto Humanus (EESIH). Esta cooperación simbiótica permite el desarrollo de actividades conjuntas de los alumnos de enseñanza superior formal de ESE-IPC y de los alumnos de la EESIH. En el ámbito de esa simbiosis, fue creado el Club de Expresión Dramática "Os Putos" en el seno de la EESIH.

\section{L'OS PUTOS}

El Club tiene su origen en el Proyecto de innovación pedagógica SeCApS Sostenibilidad Curricular y Aprendizaje en Servicio que el primer firmante del trabajo viene desarrollando en ESE-IPC desde el año de 2015 (Ramos, 2015, 2016a, 2019; Sadio Ramos, 2016). Sus componentes fundamentales son la idea de sostenibilidad curricular, dirigiendo las actividades curriculares bajo un entendimiento integral y holístico de la educación, idea complementada con la práctica del aprendizaje en servicio, por lo que se busca la inserción sociocultural de los contenidos y actividades curriculares desarrollados por los alumnos (Sigmon, 1979, 1994; Kolb, 1984; Furco, 1996; CRUE, 2005; CER, 1994; Fernandes, 2018).

Se ha aplicado el proyecto a un número considerable de especialidades curriculares, en las cuales el alumnado viene desarrollando diversas experiencias pedagógicas de intervención sociocultural por medio de actividades expresivas (física, musical y dramática; Ramos, 2016b, 2016c; Sadio-Ramos y Ortiz-Molina, 2018; Ramos y OrtizMolina, 2019). En el ámbito del SeCApS, se llevó a cabo una experiencia pedagógica en el curso de 2014-15, que posibilitó la creación del Club en diciembre de 2015. La base ha sido la asignatura de Educación No Formal con Poblaciones Específicas de la Titulación de Teatro y Educación, integrada en el $3^{\text {er }}$ curso de la misma. El programa de la asignatura presupone que los alumnos definan y desarrollen un proyecto de intervención sociocultural dirigido a una población específica; el proyecto puede ser efectivado en el cuatrimestre en que se imparte la materia o definido para su posterior realización. En el caso de la experiencia referida, la población elegida fue el alumnado de la EESIH, personas mayores de 55 años. Se definió, en el segundo cuatrimestre de 2014-2015, un primer proyecto -Ayer, Hoy y Mañana (Melo, Cardoso, Soares y Alves, 2015) - con la memoria como inductor de las actividades a desarrollar; su operacionalización y desarrollo posterior dio origen a Ayer, Hoy y Mañana - Viene (Re)viver (Melo y Paulete, 2015), que fue realizado en el curso siguiente, en diciembre.

A esta realización, se siguió la creación, en 2016, del Club de Expresión Dramática “Os Putos", y desde entonces, sus miembros han desarrollado la práctica estética de la Expresión Dramática, dando origen a un portafolio de piezas y performances públicas, a la par que se forman personalmente y en el ámbito de la especialidad artística.

Integran el Club los siguientes miembros: Alfredo Rodrigues da Costa, Ana Carolina Branco Paulete (Formadora permanente, una de las responsables de la aplicación inicial del proyecto Ayer, Hoy y Mañana), António Coelho Tejo, António Machado Dinis, Ascensão Maria Pereira Ribeiro Neves, Fernando J. Sadio Ramos (Responsable), llídio Augusto Pereira, Jorge Manuel do Espírito Santo, José Manuel Castanheira R. da Costa (miembro honorario), Maria de Fátima Fidalgo, Maria de Jesus Fernandes Barra 
Assunção, Maria Isabel Monteiro, Maria Gabriela da Costa Carvalho, Maria Rosa Lauriano Gomes Dinis Tejo. El movimiento de entrada y salida de miembros no es muy relevante, a lo largo de estos años de actividad, para lo que contribuye la dinámica del trabajo efectuado y de las relaciones personales y grupales que se desarrollan en su transcurso.

Referir, igualmente, que este curso lectivo ya ha sido posible tomar el Club como laboratorio de formación de los alumnos de la titulación de Teatro y Educación que cursan la asignatura de Educación No-Formal con Poblaciones Específicas, que desarrollan sus proyectos con ellos como sus formandos.

El porfolio del Club tiene ya una cierta consistencia, obtenida a lo largo de estos años de actividad. Los trabajos son presentados, tanto en el ámbito interno de ESE-IPC, como en cuantos momentos culturales y académicos incluídos en congresos y simposios nacionales e internacionales. En una simple enumeración, integran ese portafolio las siguientes piezas: 2016, Há-de passar (29 de Abril, Lisboa) y M(un)dificado Miguel (15 de Junio, ESE-IPC); 2017, Sonho de uma noite de Verão (adaptación; 27 de Enero, ESE-IPC); 2017, Inquietações (28 de Junio, 16 de Octubre, ESE-IPC); 2018, A Herança perdida (16 de Mayo, ESE-IPC, 13 de Junio, Casa de los Pobres, Coimbra); 2019, Um dia no Mercado (24 de Enero, ESE-IPC). En preparación, se está definiendo una pieza mezclando momentos destacados de obras de revista que hacen parte de la cultura popular portuguesa, y que tendrá su presentación pública en junio próximo.

La última representación, Un día en el Mercado, ha integrado en su desarrollo una contribución interdisciplinar de las artes plásticas, a cargo de la tercera firmante de la comunicación. Durante su estancia Erasmus+ en ESE-IPC, se ha asociado al trabajo en curso, diseñando una presentación y un escenario digital para enriquecer y reforzar la presentación pública de la pieza.

\section{LA EXPERIENCIA INTERDISCIPLINAR}

En este apartado damos cuenta de la experiencia interdisciplinar desarrollada por medio de la asociación de la actividad del Club a la Expresión Plástica, de cuyo resultado tuvimos mucho más que un día de mercado en Portugal merced de conjunción de ambas formas de expresión.

El Aprendizaje-Servicio como metodología dentro de las aportaciones que la labor de enseñar y compartir conlleva, nos encamina a participar en propuestas para consolidar aprendizajes, colaborar en su puesta en práctica y mejorar dinámicas donde la competencia social y ciudadana sean una responsabilidad y un servicio fundamental a la sociedad. Por ello, activa dinámicas e integra a colectivos dentro de la comunidad educativa.

Con esta propuesta interdisciplinar entre Expresión Dramática y Plástica, el apoyo que se genera enriquece las relaciones de comunicación en un proyecto común y se ponen en valor varios aspectos de gran interés. Por un lado, a nivel personal, conocer el trabajo del grupo Senior, cuya actividad resulta muy relevante en el sentido de integrar a personas mayores en el ámbito docente del Instituto Politécnico de Coimbra. Se trata, por tanto, de participar en un proyecto ApS, en el que se viene trabajando desde 2015, como hemos referido anteriormente.

Una de las claves de su éxito se puede focalizar en salir del aula tradicional para seguir aprendiendo. Por ello, nos abrimos al público, a la gente de nuestro entorno, y establecemos unas relaciones intergeneracionales muy enriquecedoras. La primera 
impresión, que se puede destacar, surge al observar el desarrollo del trabajo en equipo en el que se basan los planteamientos a la hora de crear un texto dramático a partir de un tema. En las primeras sesiones se pone de manifiesto la participación activa de los participantes y cómo las deliberaciones fomentan una discusión consensuada para llegar a los acuerdos oportunos y toma de decisiones colectivas y participadas. Las propuestas individuales se plantean al grupo y entre todos valoran la pertinencia de las mismas. La presencia en cada sesión de todos los componentes con sus formadores muestra uno de los objetivos alcanzados, pues la asistencia es una constante en las diferentes sesiones. Se trata de una experiencia que aporta iniciativa, creatividad, reflexión, respeto e implicación. Estos aspectos son muy loables en una sociedad donde, a veces, dejamos de lado la experiencia de nuestros mayores. De ellos debemos enriquecernos y poner en valor su potencial y sus recursos.

Analizamos el tema, en este caso un mercado tradicional, dada la obra en la que se hallan inmersos, y a partir del espacio donde se va a representar nuestro cometido hemos de resolver dos cuestiones. La primera de ellas, a modo de introducción para contextualizar su propuesta. Para ello, preparamos un audiovisual donde se incluyen imágenes de mercados portugueses tradicionales donde lo personal, las relaciones entre vendedor y cliente son muy próximas. De esta forma, y "sin palabras", nos ubicamos en la situación en la que nos vamos a ver imbuidos por los actores y actrices. Mientras las imágenes avanzan la música instrumental, El mercat a la plaça, de Luís Llach, con su algarabía, nos introduce en la dinámica de la vida cotidiana. La proyección termina con una puerta que se abre para dar paso a la representación, seguida de un plano fijo, una ilustración de un mercado como fondo, que aporta un fondo al escenario. Una imagen en perspectiva que resuelve la contextualización y nos adentramos, con este fondo virtual, en la obra.
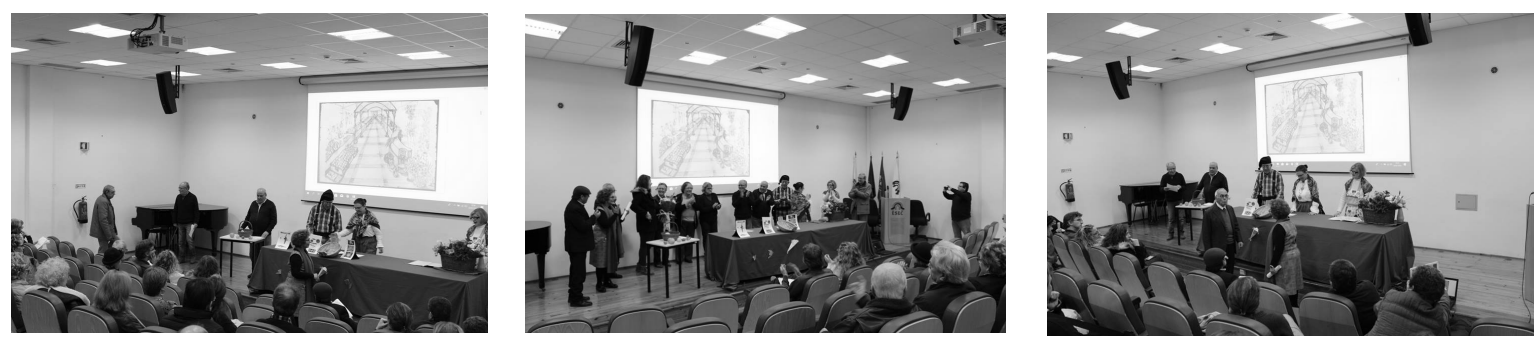

Algunas fotos de Un día en el Mercado (Cortesía IHSénior)

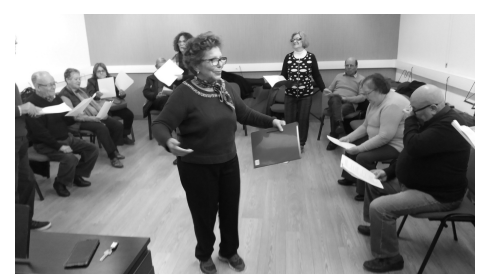

Un ensayo de la pieza (F. J. Sadio-Ramos)

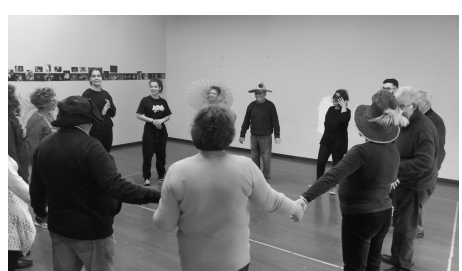

Laboratorio

(F. J. Sadio-Ramos)

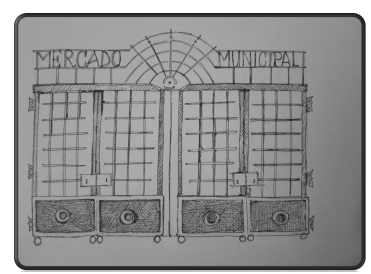

El mercado de Coimbra

(C. Martínez-Samper)

\section{CONCLUSIONES}

A lo largo de toda la experiencia vivida participamos de los resultados emanados de la utilización de una metodología de aprendizaje-servicio que ha permitido crear una 
realidad social nueva, útil y desarrolladora de un sector de la población de la comunidad. La integración de un sector de la comunidad en el ámbito de la enseñanza relaciona una formación que permite desarrollar y dar respuesta a necesidades reales. Ofrece unas dinámicas para generar un aprendizaje mutuo; se genera un observatorio social y da pie a una empatía necesaria donde mirarse cara a cara forma parte del conocimiento y del proceso. La expresión, la alegría, la satisfacción y el buen hacer generados al llevar a la práctica un proyecto colaborativo y participativo que se abre a la realidad social que nos rodea, donde el trato humano forma parte de un aprendizaje "de ida y vuelta".

El aprendizaje realizado por los miembros integrantes del Club es estudiado en el ámbito de otro proyecto de investigación y desarrollo basado en la práctica, que los primeros firmantes tienen en curso por medio de procedimientos metodológicos biográficonarrativos y en los que los aspectos de crecimiento y relación personal e interpersonal son estudiados y que son tema de otros trabajos a publicar. Se han detectado y reconocido señalados efectos de crecimiento y desarrollo personal por parte de los miembros de l'Os Putos a diversos niveles, además de la contribución sociocultural que su trabajo y actividad produce (Sadio-Ramos y Ortiz-Molina, 2019), en la línea de otros estudios sobre poblaciones de idéntico tenor (Barros, 2014; Figueira, 2004).

\section{REFERENCIAS}

Barros, G. B. de M. (2014). Teatro na Terceira Idade - possibilidades e limites de uma prática cênica (Trabalho de Projeto-Mestrado em Artes Cênicas, Faculdade de Ciências Sociais e Humanas - Universidade Nova de Lisboa). Disponible en: https://run.unl.pt/bitstream/10362/19082/1/TRABALHO\%20DE\%20PROJETO\%20\%20TEATRO\%20NA\%20TERCEIRA\%20IDADE\%20\%20POSSIBILIDADES\%20E\%20LIMITES\%20DE\%20UMA\%20PR\%C3\%81TICA\% $20 \mathrm{C} \%$ C3\%8ANICA.pdf

CER - Conference of European Rectors (1994). Carta Copernicus. The University charter for Sustainable Development. Disponible en: http://utemsustentable.blogutem.cl/files/2011/08/CARTA-COPERNICUS.pdf

CRUE - Conferencia de Rectores de las Universidades Españolas (2005). Directrices para la introducción de la Sostenibilidad en el Currículum. Disponible en: https://www.crue.org/Documentos\%20compartidos/Declaraciones/Directrices_Soste niblidad_Crue2012.pdf

Fernandes, J. L. de M. S. P. (2018). Desafios e oportunidades para a comunicação das organizações nos Objetivos de Desenvolvimento Sustentável. DEDiCA. REVISTA DE EDUCAÇÃO E HUMANIDADES, 14, 103-117.

Figueira, A. P. C. (2004) . O Palco da Vida: A expressão dramática enquanto instrumento operatório do desenvolvimento das competências sociais. Psicologia.pt, 19-02-2004. Disponible en: http://www.psicologia.pt/artigos/ver_artigo.php?codigo=A0177

Furco, A. (1996). Service-Learning: A Balanced Approach to Experiential Education. In B. Taylor and Corporation for National Service (Eds.), Expanding Boundaries: Serving and Learning (p. 2-6). Washington, DC: Corporation for National Service.

GA - General Assembly of the United Nations (2015). Resolution adopted by the General Assembly on 25 September 2015. 70/1. Transforming our world: the 2030 Agenda for Sustainable Development.

Disponible en: http://www.un.org/ga/search/view_doc.asp?symbol=A/RES/70/1\&Lang=E

Kolb, D. (1984). Experiential learning: experience as the source of learning and development. Englewood Cliffs, NJ: Prentice Hall. 
Melo, B., Cardoso, D., Soares, I. y Alves, J. (2015). Ontem, Hoje e Amanhã (Trabalho de avaliação da Unidade Curicular de Educação Não-Formal com Populações Específicas - Escola Superior de Educação do Instituto Politécnico de Coimbra). Trabajo escolar impreso.

Melo, B. y Paulete, C. (2015). Ontem, hoje e amanhã - Vem (Re)Viver [CADERNO DE EXPERIÊNCIAS ApS (Aprendizagem em Serviço) ${ }^{\circledR}$ ]. Informe presentado en el ámbito del Proyecto de innovación pedagógica SeCApS - Sostenibilidad Curricular y Aprendizaje en Servicio.

Ramos, F. J. S. (Org.) (2019). CADERNO DE EXPERIÊNCIAS ApS (Aprendizagem em Serviço ${ }^{\circledR}$. 5. ${ }^{a}$ edição. Coimbra: Fernando Ramos (Editor) ${ }^{\circledR}$. Anteriores: 2015; 2016; $2017 ; 2018$.

Ramos, F. S. (2016a). Perspectivas de Sostenibilidad Curricular - Experiencias de Aprendizaje en Servicio en Enseñanza Superior. In T. Ramiro-Sánchez, M ${ }^{a} \mathrm{~T}$. Ramiro Sánchez \& $M^{a}$ Paz Bermúdez Sánchez (Coords.), Libro de Actas del 4th International Congress of Educational Sciences and Development (p. 563). Asociación Española de Psicología Conductual (AEPC) Granada: Asociación Española de Psicología Conductual (AEPC). Disponible en: https://www.congresoeducacion.es/edu_web4/LIBRO_ACTAS_2016.pdf

Ramos, F. S. (Coord.) (2016b). Sustentabilidade Curricular - 3 experiências ApS (Aprendizagem em Serviço) em Desporto e Lazer. Póster presentado en el XIV SIEMAI® / IX ENCONTRO DE PRIMAVERA® Educación, Artes y Humanidades en una encrucijada de Culturas. Campus de Teruel, Universidad de Zaragoza, 13-16 de Abril de 2016. Incluye: a) Cortesão, B.; Vicente, C.; Freitas, M.; Silva, S.: 1. O Lazer no Trabalho; b) Antunes, A.; Likhatcheva, C.; Mendes, R.; Brandão, S.: 2. A problemática do Isolamento Social na Terceira Idade; c) Moreira, J. P. M. S.: 3. Um novo olhar - Inclusão pelo Desporto.

Ramos, F. S. (2016c). Experiências ApS no Ensino Superior: Expressão Dramática com Seniores. Póster presentado en 30 de Abril de 2016 en el "Encontro Nacional da APCEP A Educação Permanente em Tempo de Mudança: Saber para Transformar". Pavilhão do Conhecimento/ Ciência Viva, Lisboa, 29-30 de Abril de 2016.

Ramos, F. J. S. (2015). Marca Registada Nacional: CADERNO DE EXPERIÊNCIAS ApS (Aprendizagem em Serviço) ${ }^{\circledR}$ - n. ${ }^{\circ} 549165$

Ramos, F. J. S. y Ortiz-Molina, M. ${ }^{a}$ A. (2019). ExeDraSen: Expresión Dramática con Séniores. Un Proyecto I+DBP. In F. J. S. Ramos \& $\mathrm{M}^{\mathrm{a}} \mathrm{A}$. Ortiz-Molina (Coords.), $V$ Simpósio DEDiCA EDUCAÇÃO E HUMANIDADES. Sustentabilidade curricular: inovação e tradição. Livro de atas (p. 52-55). Coimbra: Fernando Ramos (Editor).

Sadio Ramos, F. J. (2016). Aprendizaje en Servicio y Sostenibilidad Curricular Experiencias de Aprendizaje en Servicio (ApS) en Enseñanza Superior. In F. S. Ramos \& M. ${ }^{a}$ A. Ortiz-Molina (2016) (Coords.), Educación, Artes y Humanidades en una encrucijada de Culturas. Atas do XIV SIEMAI - Simpósio Internacional Educação Música Artes Interculturais/ IX Encontro de Primavera. Teruel, 13-16 de abril de 2016 (p. 93-96). Coimbra: Fernando Ramos (Editor) ${ }^{\circledR}$.

Sadio-Ramos, F. J.; Ortiz-Molina, Ma . A. (2018). ContiTuna: Intervención sociocultural por medio de la práctica musical popular. Modulema. Revista Científica sobre Diversidad Cultural, 2, 7-29. Disponible en: http://revistaseug.ugr.es/index.php/modulema/article/view/6877/6340

Sigmon, R. (1994). Serving to Learn, Learning to Serve. Linking Service with Learning. Council for Independent Colleges Report.

Sigmon, R. (1979). Service-learning: Three Principles. Synergist. National Center for Service-Learning, ACTION, 8(1), 9-11. 